\title{
De la neurolobiología a la psicosociología del uso-abuso del cannabis
}

\author{
Bobes, J.*; Calafat, A.* * \\ * Catedrático de Psiquiatría. Universidad de Oviedo. \\ ** Director Revista Adicciones. Socidrogalcohol.

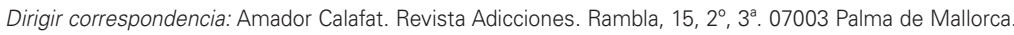 \\ adicciones@socidrogalcohol.org
}

\section{Resumen}

Los autores muestran su preocupación por la extensión creciente y la banalización del consumo y por la falta de información contrastada que está circulando, que lleva a muchas personas especialmente jóvenes a conclusiones y decisiones erróneas sobre el consumo. Se valoran positivamente todas las nuevas investigaciones tanto neurobiológicas, como psiquiátricas como sociológicas que aportan luz a esta nueva situación de consumo. Los avances neurobiológicos habidos en los últimos años y las expectativas terapéuticas que conllevan, son evaluados en términos de futuro. Se abre una visión crítica sobre la cultura que sostiene el cannabis que abren el campo sobre futuros estudios.

El impacto y los efectos, tanto deletéreos como terapéuticos de los derivados cannábicos son valorados por los coordinadores de la obra en términos de afectación de la salud comunitaria y de las personas. Asimismo, se seleccionan los componentes principales que contribuyen a fundamentar el actual debate social sobre los diversos usos del cannabis (terapéuticos, médicos, etc.).

Finalmente, se revisan los aspectos de prevención, tanto en el ámbito escolar como social y las posibilidades terapéuticas que para este campo han sido desarrolladas hasta el momento.

Palabras clave: Cannabis, epidemiología, neurobiología, prevención, tratamiento, sociología.

\section{Summary}

The authors demonstrate their preoccupation with the increasingly widespread and commonplace use of cannabis, and with the lack of contradictory information in circulation, which leads many people, particularly the young, to erroneous decisions on use. All the new research, neurobiological, psychiatric and sociological, that sheds light on this new use situation is positively evaluated. The neurobiological advances made in the last few years and the therapeutic prospects which they bring with them are evaluated in terms of the future. There is a critical look at the culture that sustains cannabis, opening up the field to future studies.

The co-ordinators of the work evaluate both the deleterious and therapeutic impact and effects of cannabis derivatives in terms of the effect on community and personal health. In addition, there is a selection of the principal factors that contribute to basing the present social debate on the diverse uses of cannabis (therapeutic, medical, etc.).

Finally, prevention aspects are reviewed within both the scholastic and social spheres, and the therapeutic possibilities which have been developed in this field up to the present time.

Key words: Cannabis, epidemiology, neurobiology, prevention, treatment, sociology. 


\section{INTRODUCCIÓN}

E uso de la cannabis sativa tanto como sustancia psicotropa como con finalidades industriales es muy antiguo (Ramos, Fernández-Ruiz , 2000b). Desde hace ya años el cannabis se ha convertido en la droga ilegal más consumida con diferencia en nuestro y otros países, por lo que no deja de llamar la atención la escasa literatura científica, que aborda los problemas que se derivan del uso de dicha sustancia, a pesar de que se han hecho importantes progresos en los últimos años como es el descubrimiento de un sistema cannabinoide endógeno. Mientras, otras drogas como la heroína o la cocaína están recibiendo mucha mayor atención en la literatura tanto profesional como en los medios de comunicación. Diversos fenómenos sociales han hecho que aminorase toda la alarma social vinculada al uso del cannabis, consiguiendo una imagen social de droga que no causa problemas. Las cosas parecen estar cambiando $y$, tal como dice, tras tres décadas de un uso en expansión de esta sustancia, estamos presenciando la maduración sobre la discusión en algunos temas relacionados con el cannabis. Estas discusiones están todavía la mayor parte de las veces llenas de pasión, de connotaciones políticas o ideológicas, lo que lleva necesariamente a que las posturas están en muchas ocasiones demasiado confrontadas. Con esta monografía tratamos de aportar la mayor información actual contrastada para poder avanzar por este camino de una forma seria, rigurosa y responsable, separando, en la medida de lo posible los valores de las opiniones y posturas de los datos científicos, lo cual, a día de hoy, sigue siendo todavía una misión difícil. Sin embargo, los últimos años han sido fecundos en cuanto a aportación de nuevos datos contrastados.

\section{EPIDEMIOLOGÍA.}

Es precisamente la extensión de su uso el hecho que más poderosamente llama la aten- ción sobre esta droga (Bobes et al. 2000). Se estima que entre un 7-10\% de la población mundial podrían ser consumidores regulares de la sustancia, la mayoría de ellos en regiones donde su uso es ilegal. La droga ilícita más consumida en la Unión Europea es también el cannabis, estimándose en aproximadamente más de 40 millones el número de personas que lo han usado, y en al menos 12 millones el número de personas que lo han usado en el último año. Es decir, aproximadamente el $16 \%$ de la población de la Unión Europea de edades comprendidas entre los 15 y los 64 años habrían consumido cannabis alguna vez en su vida, y cerca del $5 \%$ lo habrían consumido en el último año.

España es uno de los países lideres en este consumo. Según datos de la Encuesta Domiciliaria sobre Consumo de Drogas de 1997, un $21.7 \%$ de los españoles de edad comprendida entre 15 y 65 años refieren haber consumido la sustancia alguna vez en la vida, un $7.5 \%$ en el último año, un $4 \%$ en el mes previo y un $0.9 \%$ lo hacía diariamente (Plan Nacional sobre Drogas, 1998), aunque la encuesta del 99 indicaría un cierto descenso pasando a un $6,8 \%$ los que la habrían probado durante el último año.

Cuando se comparan los datos de la Encuesta de Población sobre el consumo de cannabis por los adultos de los Estados miembros de la Unión Europea nos encontramos que España está a la cabeza del "ranking", únicamente superada por Dinamarca y el Reino Unido. El $22 \%$ de todos los adultos españoles y el $32 \%$ del subgrupo de adultos jóvenes han consumido cannabis alguna vez en su vida.

Este amplio uso no es obviamente estático y ha sufrido amplias oscilaciones a lo largo de los años, lo que hace pensar en la importancia de las cuestiones socioculturales tanto en lo que supone incrementos como en lo que podrían ser descensos en su uso. En Europa durante la década de los 80 el consumo se habría estabilizado y es en la década de los 90 en la que se vuelve a experimentar nuevamente un gran incremento (EMCDDA, 1998). Por tanto, y esto es importante desde el 
punto de las estrategias políticas y preventivas, no estamos ante un fenómeno estable, sino ante un proceso sociocultural influenciable en uno u otro sentido.

Este amplio uso tiene por descontado diversas causas. Pero sin lugar a dudas hay que atribuir una importancia especial al extenso y complejo entramado sociocultural que lo sostiene. Se trata de una cultura rica y en plena evolución, que se está apropiando paulatinamente de una serie de valores pertenecientes hasta ahora a otras dinámicas culturales, generando una situación muy peculiar y cada vez más diversa en contenidos, que acaba sirviendo para justificar el consumo de cannabis. No obstante esta sub-cultura particular es de momento una cuestión más adoptada por las consumidores de más edad, mientras que los más jóvenes son unos consumidores más compulsivos e indiscriminados de drogas de fin de semana, sustentados por una ideología de consumo (de tiempo libre, de diversión, de sexualidad, etc...).

\section{SISTEMA CANNABINOIDE ENDÓGENO.}

Tres fechas marcan el conocimiento de los aspectos neurobiológicos del cannabis. Se trata de 1964 con el aislamiento del tetrahidrocannabinol (THC), 1990 con el descubrimiento de los receptores cannabinoides en el cerebro y 1994 con el descubrimiento del ligando endógeno natural, la anandamida. Junto con estos cannabinoides endógenos del cerebro y los vegetales de la planta cannabis sativa existen además los cannabinoides sintéticos. Toda esta parte química, farmacológica y neurofisiológica recibe un importante tratamiento dentro de esta monografía por ser la parte más novedosa y desconocida para muchos de los profesionales y donde se está investigando más en estos momentos ;, , )

La distribución cerebral de los endocannabinoides y de los receptores cerebrales hacen pensar en un papel de modulación del sistema cannabinoide sobre el sistema dopaminérgico, pero se le presumen y se están investi- gando muchas otras funciones. Este receptor está presente a lo largo de toda la escala vertebrada, con un patrón de distribución que se ha conservado a lo largo de la evolución. En humanos, su gen se encuentra en la región q14-q15 del cromosoma 6, presentando una homología del 97,3\% con el de rata.

Los receptores cannabinoides cerebrales están situados próximos a otras neuronas pertenecientes a muchos circuitos cerebrales, incluido el sistema de recompensa cerebral. De acuerdo a ) la administración crónica de cannabinoides induce neuroadaptaciones en el circuito de recompensa que son idénticas a las inducidas por las principales drogas de abuso y que se pueden poner de manifiesto mediante el cese de la administración de estos compuestos. Los cannabinoides actúan sinérgicamente con el sistema opioide lo que les permite actuar como factores de riesgo en el desarrollo de la conducta adictiva. Según estos autores, la existencia de una interacción opioide -cannabinoide permitirá abrir nuevas puertas terapéuticas para la adicción a la heroína y el alcohol.

\section{IMPACTOY EFECTOS DEL CANNABIS.}

Son más de 400 las sustancias identificadas en el cannabis de las que alrededor de 60 son cannabinoides, entre los que se encuentra el más importante que es el THC. En un informe realizado por un grupo de expertos para la ) en 1995 se afirmaba que la repercusión del cannabis sobre la salud en términos cuantitativos era todavía difícil de establecer debido a la falta de estudios epidemiológicos convincentes. Además los posibles problemas relacionados con el uso de cualquier droga están muy relacionados con el contexto cultural y social, el contexto económico y político, la disponibilidad, las formas de presentación y su concentración, las formas de administración, la frecuencia de uso y el estilo de vida asociado. Otra dificultad adicional, según dicho informe, para conocer los efectos del cannabis residiría en que muchos consumidores hacen un uso simultáneo de varias dro- 
gas, y el riesgo de usar varias drogas a la vez no tiene porque ser necesariamente la suma de los riesgos individuales. En definitiva, no es posible todavía llegar a una comparación definitiva entre los problemas derivados del uso del cannabis y los producidos por el tabaco y el alcohol, por la insuficiencia de información y porque el número de consumidores regulares de cannabis es netamente inferior al de los otros productos. Dicho informe acaba indicando la necesidad de profundizar en la investigación en áreas como la clínica y la epidemiológica, la química y la farmacología, los efectos terapéuticos y los efectos que tiene sobre la salud el cannabis.

La falta de estudios y sobre todo el desconocimiento -interesado o no- de los estudios existentes ha facilitado la extensión de diversas ideas erróneas sobre el cannabis entre la población general. No deja de sorprender la fuerza con la que se ha introducido en el imaginario social durante los últimos años la idea de la falta de problemas ligados al consumo de marihuana. De la misma forma que existen organismos e instituciones que trabajan a favor de las restricciones en el uso del cannabis, también existe un potente y organizado movimiento pro cultura del cannabis Calafat et al. (2000b) que ha conseguido que sus mensajes calen profundamente en amplias capas de la población, incluidos amplios sectores de profesionales, potenciándose la idea del cannabis cómo una droga recreativa que "no" causa problemas y con posibles utilidades terapéuticas y que sólo el miedo y el desconocimiento pueden explicar su situación legal y social actual. En efecto los ciudadanos adultos de una sociedad determinada en su conjunto deben poder decidir sobre el estatuto que tiene una determinada droga desde el punto de vista legal o en su vida diaria. Pero es importante también que exista información pertinente y de calidad y que no se produzcan informaciones sesgadas por uno $u$ otro lado.

A pesar de la existencia de numerosos mensajes que hacen referencia a la inocuidad del consumo del cannabis, los datos existentes en el momento actual ponen de manifies- to que el consumo de esta sustancia no está exento de problemas (Quiroga 2000a y 2000b). Así, aunque el consumo ocasional de cannabis no acarrea consecuencias orgánicas graves y su empleo habitual lo hace en pocas ocasiones, se han descrito diversas complicaciones en diferentes órganos y sistemas (aparato respiratorio, sistema inmune, función gonadal y reproductora, efectos sobre el feto, etc.) asociadas a su uso regular. Por otra parte, no hay que olvidar que en los servicios de urgencias hospitalarios son frecuentes las consultas por complicaciones psicopatológicas agudas (crisis de angustia, estados de ansiedad) derivadas de su utilización. De igual modo, se han descrito y observado en la clínica otros cuadros de índole psiquiátrica tales como episodios de flashback, trastornos psicóticos y cuadros confusionales asociados al consumo de esta sustancia. La información referente a la asociación entre el consumo de cannabis y la aparición de trastornos psiquiátricos es parcialmente controvertida. En el momento actual se da gran valor al papel que una predisposición (vulnerabilidad) previa pudiera tener en el desencadenamiento de los diferentes cuadros psicopatológicos, fundamentalmente en las psicosis, ya que existen hallazgos que indican que la capacidad de generar cuadros psicóticos es muy superior en los que consumen derivados opiáceos, y, por tanto, se acepta en la literatura que el uso de estimulantes dopaminérgicos, como el cannabis o las anfetaminas, facilitan la aparición de trastornos mentales y/o del comportamiento. No obstante, se desconoce hasta que punto el cannabis actúa como desencadenante, es una consecuencia o simplemente coincide con la psicopatología.

Otro aspecto de interés relacionado con efectos a medio y largo plazo del uso/abuso de cannabis, es el denominado síndrome amotivacional, caracterizado por apatía, desinterés, incapacidad para desarrollar planes futuros, empobrecimiento afectivo, abandono del cuidado personal, inhibición sexual, etc., todo ello acompañado de una serie de alteraciones psicomotoras como: disminución de los reflejos, parquedad de movimientos, lentitud de 
desplazamientos, etc. La consecuencia directa de dicho síndrome sería una falta de voluntad propia y las indirectas un descenso en el rendimiento académico o laboral, deterioro de las habilidades comunicativas y retraimiento social. La atribución causal de este síndrome sigue sin estar clara y se discute si es consecuencia del consumo de cannabis o más bien se trata de una especial tendencia previa a la pasividad de los sujetos dependientes de esta droga. Resultan necesarios estudios longitudinales bien diseñados podrán establecer de una forma definitiva la aparición o no de este trastorno en una población determinada.

Un daño indirecto derivado del consumo de cannabis es la accidentabilidad en carretera. Actualmente se dispone de suficiente información que pone de manifiesto que el cannabis puede interferir con la destreza y otras habilidades propias de la conducción de automóviles. Además, se ha constatado que una importante proporción de jóvenes realizan desplazamientos en automóvil a altas horas de la madrugada, no sólo bajo los efectos de cannabis, sino también de otras drogas como alcohol y psicoestimulantes, lo cuál puede contribuir a las elevadas tasas de accidentabilidad detectadas en nuestros jóvenes, ya que entre los 18 y 34 años de edad se concentra el $40 \%$ de los casi 5.000 muertos que cada año se cobra la carretera y los análisis toxicológicos revelan la presencia de drogas en una proporción nada desdeñable de dichos jóvenes. En esta área no es conveniente pasar por alto el incremento de potencial tóxico al utilizar los derivados cannábicos juntamente con otras drogas, al igual que se ha demostrado con el uso de derivados de la cocaína y alcohol, dando lugar a otros productos más tóxicos como la metil-ecgonina.

\section{EL DEBATE SOBRE FACILITACIÓN DEL USO DEL CANNABIS.}

Que duda cabe que el cannabis no debe ser alineado por sus efectos con otras drogas como la heroína o la cocaína, pero cosa muy distinta es referirse a dicha droga como exen- ta de cualquier problema, cuando ya existe bastante información contrastada y con base empírica sobre sus efectos sobre la salud física y psíquica (Quiroga 2000a, b). Pero, ¿Cómo no vamos a preocuparnos acerca del cannabis cuando en una muestra de 2.617 jóvenes seleccionados en zonas de diversión de fin de semana en 9 ciudades europeas nos encontramos con un $10 \%$ de consumidores diarios y en otra muestra de 1.333 jóvenes de 5 ciudades españolas este porcentaje se eleva hasta el 15,8\%, cuando por otro lado sólo encontramos dentro de esta muestra un $8,3 \%$ y un $6,5 \%$ respectivamente de consumidores diarios de alcohol (Calafat et al. 2000a).

Especialmente preocupante es la repercusión que tiene la banalización del uso del cannabis por los adolescentes, donde precisamente se está experimentando un mayor incremento del uso y donde menos información rigurosa existe sobre los efectos y donde existe una menor percepción del riesgo que asumen con su consumo. Conviene, entendemos nosotros, diferenciar claramente el uso que hace del cannabis un adulto responsable, del uso indiscriminado frecuentemente junto a otras drogas, que hacen los adolescentes.

En un estudio de Johnston LD y O'Malley PM (1998), aprovechando el seguimiento anual que se hace del consumo de diversas drogas desde 1976 a 1996 en USA, encontró que diversos factores estaban relacionados con la evolución anual en el consumo de cannabis (y otras drogas) como podían ser el cumplimiento con los estudios, implicación en actividades antisociales, etc., pero son la percepción del riesgo o la desaprobación de una determinada droga lo que determina más exactamente año tras año los cambios en el consumo. Preocupa en este sentido el descenso de la percepción del riesgo en general entre la población española, los jóvenes españoles en particular y sobre todo el descenso en la percepción de riesgo detectada en la Encuesta escolar de 1998 (Plan Nacional sobre drogas, 2000) entre los adolescentes 
de 14 y 15 años en relación con las encuestas anteriores.

Estamos ante un debate vivo que afecta a diversos aspectos del cannabis como son las cuestiones legales, sus efectos, sus cualidades terapéuticas, la existencia de dependencia, .... Es un debate que va más allá de una cuestión periodística y de hecho ha entrado de lleno también en los medios profesionales, aunque en este caso las posturas son bastante más matizadas. Son frecuentes las posturas maniqueas y esto está llevando a una dificultad para avanzar de forma racional y científica en dicho debate. Un debate similar (Calafat, 1997) se produjo a finales de los 80 y principios de los 90 alrededor del MDMA, conocido como éxtasis. Una posición mantenía la inocuidad casi absoluta de dicha sustancia, defendiendo incluso que se derivaban beneficios de su uso pues disminuía el uso de otras sustancias como el alcohol, mientras que la otra postura -sustentada especialmente por la prensa amarilla inglesadefendía que era una droga que mataba. Junto con estas cuestiones que giran alrededor de la bondad o maldad de sus efectos, existía también todo un entramado cultural (que genéricamente podemos denominar house, rave, techno o bacalao) que definía los contenidos y el comportamiento de muchos adolescentes y jóvenes de los 90 y de la actualidad y donde el consumo de 'drogas de diseño' se convertía en una cuestión central. Fue curioso observar como un sector relativamente importante de profesionales de las drogodependencias (especialmente en Holanda, Inglaterra, Alemania,...) se apuntaba a defensa del éxtasis y de la cultura techno (Fromberg, 1998), poniendo incluso en marcha una visión preventiva particular basada en el análisis del contenido de las pastillas, pues, según estos profesionales, el peligro estaría no tanto en el consumo de la sustancia en si misma -que por aquellos tiempos, a falta de estudios, se creía relativamente segura- sino en las posibles adulteraciones. El descubrimiento de sus efectos sobre el sistema nervioso, una mejor recogida de datos clínicos y algunos estudios epidemiológicos que des- montaron la visión idealizada del consumidor de éxtasis (no combinación con otras drogas, preocupado por la prevención de problemas,... ) (Calafat et al. 1998, 1999) e hicieron desaparecer la polémica. Actualmente, gracias al mejor conocimiento de la situación, ha desaparecido de los medios de comunicación y de los debates profesionales esta defensa soterrada o abierta del éxtasis, ha aumentado la percepción del riesgo sobre el éxtasis y, posiblemente como consecuencia de esta visión más negativa de la sustancia se ha frenado su expansión imparable hasta hace unos años en los países europeos, incluido España. Pero, esta reconducción de los hechos no ha impedido a tiempo que el éxtasis llegara a convertirse en su momento en una droga de amplio consumo, lugar que todavía sigue ocupando.

¿Cuáles son los puntos calientes de este debate actual sobre el cannabis? Básicamente la inocuidad del uso de la marihuana (dependencia, síndrome de abstinencia, psicosis cannábica, síndrome amotivacional, efectos sobre la conducción,...), su importancia en la escalada hacia otras drogas, sus usos terapéuticos (en el cáncer, glaucoma,...) y como consecuencia de todo ello la modificación del tratamiento legal.

La aparición de más información científica está forzando -o debería forzar- a todas las partes implicadas a moderar sus argumentos y posiciones. Hay cuestiones que deben tenerse en cuenta ineludiblemente como son la mayor concentración en producto activo de la marihuana actual (pudiendo llegar hasta al $20 \%$ de su contenido en THC), las evidencias crecientes sobre su influencia por ejemplo sobre el aparato respiratorio, su papel en precipitación o exacerbación de episodios psicóticos, la producción de dependencia (aunque esta sea con un menor componente físico), su relación con la conducción... Aunque siempre sacar conclusiones no será una cuestión fácil, pues, por poner un ejemplo, mientras que es un hecho que en estudios controlados el cannabis afecta la conducción y no sólo en los momentos de intoxicación (Chesher G, 1995; Quiroga M, 2000a), no es una extrapo- 
lación automática su influencia negativa sobre la conducción en condiciones normales, que necesita más investigación.

El papel del cannabis en la escalada de consumos que hacen muchos jóvenes es uno de los hitos de este debate (Vázquez F Becoña E, 2000). Es un hecho que el consumo de cannabis precede el uso de sustancias más potentes y peligrosas, pero también es cierto que sólo una minoría de consumidores de cannabis terminan utilizando otras drogas más nocivas. Es el cannabis la puerta de entrada según la hipótesis secuencial establecida por (Kandel DB et al. 1992) o bien dicho consumo de cannabis y luego de otras drogas es más bien la expresión de un "síndrome común" (Jessor R et al. 1980) según el cual hay ciertas personalidades desviantes que acaban generando una serie de actividades antisociales y el que unas conductas precedan a otras es una cuestión básicamente de la edad. Muy posiblemente la realidad del consumo sea una mezcla de los dos modelos que interaccionan.

Las cuestiones políticas también son un tema central en este debate. ¿Cuál ha sido la influencia de modelos tolerantes o de modelos con una mayor carga de represión sobre el incremento o el descenso del uso-abuso? ¿La mayor liberalización haría desaparecer el mercado negro o por el contrario haría aumentar el número de consumidores? No hay demasiados estudios que aborden estas cuestiones con una metodología adecuada y ajenos, en lo posible, a posicionamientos ideológicos extremos. En líneas generales se podría pensar que las políticas oficiales tienen menos influencia de lo que se podría suponer sobre los consumos, entre otras cosas porque a veces no es el espíritu de la ley el que se aplica (Reuband, 1995). Posiblemente las diferencias de consumo, como concluye un estudio que compara las variaciones regionales entre Holanda y Alemania en el consumo de cannabis y alcohol, se deban más a estilos de vida de los jóvenes, que varían dentro de un mismo país, más que a las políticas generales de cada país o región (Plomp et al.1996). En todo caso toda esta cuestión no se aborda expresamente en esta monografía, aunque si que se abordan los aspectos legales del cannabis en España (Herrero S, 2000) y las técnicas de laboratorio para su detección (Balcells M, 2000)

\section{USO MÉDICO.}

Es este un campo todavía reciente pero sobre el que existe un gran interés y sobre el que se está trabajando. El uso médico del cannabis y de sus componentes activos forma también parte del núcleo de la polémica que mantienen unos y otros respecto a esta sustancia. Aunque estrictamente hablando la capacidad terapéutica de uno de los 60 cannabinoides activos que contiene la marihuana en una enfermedad o síntoma determinado no tiene porque implicar la bondad o maldad de su consumo a efectos recreativos. De hecho existen cannabinoides sintéticos que emulan los efectos de los cannabinoides naturales o que incluso su eficacia es mucho mayor. Sin embargo dentro de la estrategia pro legalizacionista si que se ve como un camino que puede favorecer esta meta.

En los últimos años se han presentado un cierto número de evidencias científicas acerca de las propiedades terapéuticas de los cannabinoides como pueden ser analgesia, disminución de la presión intraocular, efecto antiemético en vómitos inducidos por quimioterapia antineoplásica, propiedades relajantes musculares en diversas enfermedades como esclerosis múltiple, traumatismos medulares y alteraciones del movimiento. Además, algunas aportaciones recientes indican otros posibles usos de estas sustancias como neuroprotectores (en modelos animales de enfermedades neurodegenerativas e isquemia cerebral), antiasmáticos y anticonvulsivantes. Más recientemente, algunos compuestos naturales y agentes sintéticos agonistas de receptores CB han demostrado efectos antineoplásicos in vivo e in vitro. Se necesitan más estudios clínicos con el fin de establecer qué dosis, vías de administración son las más adecuadas en cada 
caso, así como el balance entre beneficio y riesgo comparando los cannabinoides con otras estrategias terapéuticas (Lorenzo P, Leza JC, 2000). En algunos países existen condiciones legales que permiten avanzar en estas investigaciones y es de esperar que los próximos años sean fecundos en este sentido. En 1997 la British Medical Association publicó su informe "Therapeutic Uses of cannabis" instando a la puesta en marcha de "investigaciones controladas para evaluar el potencial terapéutico del uso del cannabis" al que sigue otra publicación del Institute of Medicine americano titulada "Medical Use of Marijuana". En síntesis, podemos admitir que las relaciones de estos usos terapéuticos con su situación legal con vistas al consumo recreativo no son en principio directas -algunas de las investigaciones en marcha utilizan los cannabinoides sintéticos- y desde un punto de vista estricto son dos situaciones totalmente diversas, pero que duda cabe que de consolidarse esta vertiente terapéutica del cannabis o de los cannabinoides, ello tendría una influencia sobre la visión social de este producto.

\section{QUIENES SON LOS USUARIOS DEL CANNABIS.}

Al tratarse de una droga cuyo uso es tan extendido cabe esperar una gran variedad de gente que lo consume. En principio son los más jóvenes quienes más lo utilizan en la actualidad, aunque un porcentaje relativamente notable de adultos lo ha fumado o lo sigue utilizando en la actualidad. Esta cuestión de la edad es clave a la hora de describir estereotipos de consumidores. No hacen el mismo uso, ni consumen posiblemente por las mismas razones un adulto de 40 o 50 años, mucho más identificado con la cultura del cannabis (Calafat et al. 2000b), que un joven o un adolescente que sale todos los fines de semana hasta altas horas de la noche, que al mismo tiempo abusa del alcohol y de otras drogas (especialmente éxtasis y cocaína) y cuyo punto de referencia es la cultura Techno o bacalao (Calafat, 2000). Aunque los consumidores de cannabis presentan características de personalidad diferenciales respecto a los no consumidores según diversas pruebas como el Cuestionario de Personalidad de Eysenck para Adultos o la Escala de Búsqueda de Sensaciones de Zuckerman (González MP et al. 2000), creemos que es también la edad del consumidor y su estilo de vida determinan la forma de consumo de cannabis, su consumo concomitante con otras drogas incluido el alcohol, o las consecuencias sobre la conducta o el físico del consumo.

\section{LA PREVENCIÓN.}

El consumo de cannabis se ha convertido en muchos países en un fenómeno social y cultural relevante, muy asociado a diversos estilos de vida. La extensión de su uso en occidente depende justamente de esta asociación profunda a elementos socioculturales. Y en los años 60 fue su asociación a la cultura hippie lo que ayudó a afianzar el consumo masivo que desde entonces han venido haciendo los jóvenes. Aunque actualmente ya no se puede afirmar que el cannabis no produce dependencia, es cierto también que su extensión masiva depende menos de la creación de una dependencia física y más del papel sociocultural que desde hace unas décadas soporta el cannabis. En este sentido la prevención del cannabis (Amengual M, 2000, ) presenta importante retos pues imaginar una política preventiva basada exclusivamente en la actuación en el ámbito escolar resultaría insuficiente. Conseguir cambios sociales profundos en la percepción de riesgos del uso y de los usuarios del cannabis es necesario. Es fundamental para lograr que disminuya el consumo de cannabis recorrer el camino inverso del que se ha recorrido hasta ahora, es decir disminuir la tolerancia social, aumentar la información sobre los efectos negativos de esta droga, contrarrestar la presión de la cultura pro cannabis, descubrir y luchar contra los intereses económi- 
cos importantes que sostienen el consumo,... Se trata de seguir los mismos pasos que se han seguido y se siguen en la lucha contra el tabaco. La máxima dificultad reside en cómo conseguir la complicidad de los medios de comunicación que están siendo una de las piezas claves en desmontar la estrategia pro tabaco.

Otro aspecto importante es cómo intervenir en el entramado sociocultural que sostiene el cannabis y las otras drogas recreativas. Lo recreativo se ha erigido en un valor positivo de primer orden resultando difícil introducir mensajes preventivos cuando todo el mundo tiende a ver las actividades lúdicas como exentas de peligro. Las drogas recreativas, entre las que el cannabis es la más importante junto con el alcohol, tienden a ser vistas en positivo, como una tecnología facilitadora de los logros recreativos. Las polémicas alrededor de la prohibición o legalización del cannabis desvían energías y retrasan la visión del uso del cannabis como un problema de salud pública y la puesta en marcha de medidas preventivas.

\section{TRATAMIENTO.}

Para muchas personas de la calle e incluso para la mayoría de usuarios de cannabis puede parecer extraño que se hable de tratamiento en relación con el cannabis, ante la idea extendida de que el cannabis no produce problemas. Pero uno de los datos que nos llama la atención es la creciente frecuencia con que los consumidores de cannabis acuden a los centros de la red pública española de tratamiento de toxicómanos. En el informe número 3 del Observatorio Español sobre Drogas (Plan Nacional sobre Drogas, 2000) se recogen los datos sobre tratamiento de 1998 de 478 centros que admitieron a tratamiento un total de 54.338 personas. Junto a la constatación de un descenso en la admisión de heroinómanos - aunque todavía suponen un $80,2 \%$ - y de una subida rápida durante los últimos años de la atención a los cocainómanos que suponen para 1998 el $11,3 \%$, tenemos que el cannabis ocupa el tercer lugar con un 5,3\%, porcentaje que está creciendo desde hace unos años. Si consideramos que los consumidores de cannabis no se identifican con el estereotipo de drogadicto, no se puede esperar de ellos que acudan fácilmente a los centros de tratamiento de tratamiento público. También no debe ser menos cierto que un porcentaje indeterminado de estas demandas de tratamiento no son atribuibles a verdaderas peticiones, sino que son personas que acuden mandados por la Autoridad Gubernativa que les ha impuesto una sanción administrativa por estar consumiendo dicha sustancia en un lugar público. Sea cual sea la proporción real de consumidores de cannabis que acuden a tratamiento de su dependencia o de alteraciones relacionadas con su uso, es un hecho constatable para todos los que hacemos clínica que con una mayor o menor frecuencia este tipo de consumidores acuden a nuestras consultas en busca de ayuda.

La dependencia física es débil, en comparación a otras drogas de abuso. La interrupción del consumo intenso y prolongado del cannabis puede producir síntomas intensos aunque relativamente inespecíficos como: desasosiego, irritabilidad, agitación y a veces insomnio, temblores, anorexia, sudoración profusa y náuseas. Además se observa un aumento "rebote" de la duración de la fase REM del sueño. Por tanto, puede decirse que el cannabis induce una dependencia fundamentalmente de tipo psicológico. Estos síntomas desaparecen al volver a consumir cannabis. En general, los consumidores exclusivos de dosis moderadas pueden abandonar el hábito de fumar cannabis con cierta facilidad, siendo aún raros los casos de demanda de ayuda en relación con el uso de cannabis, aunque como ya se ha comentado ocupan la tercera posición (por detrás de heroína y cocaína) en relación a este hecho.

El abordaje terapéutico del abuso de cannabis (Solé, 2.000) ha sido objeto de una atención más bien escasa, no existiendo estudios sistemáticos acerca del mismo. Se 
ha descrito la posible utilidad de fármacos, que incrementan la actividad noradrenérgica (cuya reducción se asocia al consumo crónico), como la desipramina, reboxetina y la tirosina podrían estar indicados para la interrupción del consumo o la prevención de recaídas, si bien estos datos han sido insuficientemente confirmados. En lo que a intervenciones psicológicas se refiere, no existen estudios acerca de cuáles pueden resultar más eficaces para el tratamiento de la dependencia a esta sustancia, si bien éstas deberían ir dirigidas a concienciar al sujeto de los riesgos del consumo y proporcionarle objetivos alcanzables que conlleven su disminución o cese. Un aspecto que consideramos de gran relevancia son los cada vez más elevados índices de policonsumo observados entre los consumidores, especialmente entre las poblaciones más jóvenes. En este sentido creemos fundamental la realización de abordajes terapéuticos que tengan presentes los diferentes consumos concomitantes, a la hora de emprender el tratamiento de pacientes que acuden solicitando ayuda por consumo de otras drogas como heroína, cocaína u otros psicoestimulantes.

\section{BIBLIOGRAFÍA}

Amengual M (2000) Enfoque preventivo del uso y abuso de cannabis y problemas asociados. Adicciones 12, supl 2.

Balcells M (2000) Toxicología del cannabis. Adicciones 12, supl 2.

Bobes, J., Bascarán MT, González MP Saiz PA (2000) Epidemiología del uso/abuso de cannabis. Adicciones 12.

Calafat A, Fernández C, Becoña E, Gil E, Juan M Torres MA (2000a) Consumo y consumidores de cannabis en la vida recreativa. Adicciones 12.

Calafat A, Juan M, Becoña E, Fernández C, Gil E Llopis JJ (2000b) Organización y estrategias de la cultura pro cannabis. Adicciones 12.

Calafat, A. (1997) La Representación Social de las Drogas de Diseño en Europa. En: XXIV Jornadas Nacionales Socidrogalcohol. Libro de
Actas, pp. 39-61. Valencia: Generalitat Valenciana.

Calafat, A., Bohrn, K., Juan M, Kokkevi A, Maalsté, N. et al (1999) Night life in Europe and recreative drug use. Sonar 98., Palma de Mallorca: IREFREA.

Calafat, A., Stocco, P., Mendes, F., Simon, J., van de Wijngaart, G., Sureda, P. et al (1998) Characteristics and Social Representation of Ecstasy in Europe., Palma de Mallorca: IREFREA.

Chesher G (1995) Cannabis and road safety: an outline of the research studies to examine the effects of cannabis on driving skills and actual driving performance. In: Anonymous The effects of drugs (other than alcohol) on road safety, pp. 67-96. Melbourne: Government Printer.

EMCDDA (1998) Annual report on the state of the drugs in the European Union, 1998 edn. Lisboa: EMCDDA.

Farrel, M. (1999) Cannabis dependence and withdrawal. Addiction 94, 1277-1278.

Fromberg, E. (1998) Réduction des dommages et ecstasy. Interventions 64, 26-37.

González MP, Saiz PA, G-Quirós M López JL (2000) Personalidad y uso-abuso de cannabis. Adicciones 12.

Herrero S (2000) El cannabis y sus derivados en el derecho penal español. Adicciones 12, supl 2.

Jessor R, Chase JA Donovan JE (1980) Psychosocial correlates of marijuana use and problem drinking in a national sample of adolescents. Am.J. of Public Health 70, 604-613.

Johnston LD O'Malley PM (1998) Explaining recent increases in student' marijuana use: impact of perceived risks and disapproval, 1976 through 1996. American J. of Public Health 887-892.

Kandel DB, Yamaguchi K Chen K (1992) Stages of progression in drug involvement from adolescence to adulthood: further evidence for the Gateway Theory. J.of Studies on Alcohol 53, 447-457.

Leza JC Lorenzo P (2000) Efectos farmacológicos de los cannabinoides. Adicciones 12, supl 2.

Lorenzo P Leza JC (2000) Utilidad terapéutica del cannabis y derivados. Adicciones 12, supl 2.

Merino PP (2000) Vieja historia del cannabis y recientes prácticas preventivas en Europa. Adicciones 12, supl 2. 
Navarro, M. Rodríguez de Fonseca, F. (2000) Cannabinoides y conducta adictiva. Adicciones 12, supl 2.

OEDT (1999) Informe anual sobre el problema de la drogodependencia en la Unión Europea. Bélgica: OEDT.

Plan Nacional sobre Drogas (1998) Memoria 1998. Madrid. Ministerio del Interior.

Plan Nacional sobre Drogas (2000) Informe $n^{\circ} 3$. Madrid. Ministerio del Interior.

Plomp, H.N., Kirschner, W., Van der Hek, H. (1996) Explanation of national variations in alcohol and cannabis consmption. A comparative study in a Dutch and adjoining german region. European J.Public Health 6, 118-125.

Quiroga M (2000a) Cannabis: efectos nocivos sobre la salud física. Adicciones 12, supl 2.

Quiroga M (2000b) Cannabis: efectos nocivos sobre la salud mental. Adicciones 12, supl 2.
Ramos JA, Fernández Ruiz J (2000a) Sistema cannabinoide endógeno: ligandos y receptores acoplados a mecanismos de transducción de señales. Adicciones 12, supl 2.

Ramos JA, Fernández Ruiz J (2000b) Uso de los cannabinoides a través de la historia. Adicciones 12 , supl 2.

Rodríguez de Fonseca, F. Navarro, M. (2000) Adicción y sistema cannabinoide endógeno: papel del receptor para cannabinoides CB1 en la fisiología de las neuronas dopaminérgicas mesotelencefálicas. Adicciones 12.

Solé Puig J (2000) Tratamiento del paciente cannábico. Adicciones 12 supl 2.

Vázquez F, Becoña E (2000) Factores de riesgo y escalada cannabinoide. Adicciones 12, supl 2.

WHO (1997) Cannabis: a health perspective and research agenda. pp.1-45. Geneva: WHO. 